\title{
Ajuste de las curvas tensión-deformación a alta temperatura de un acero microaleado $\mathrm{Nb}-\mathrm{Ti}^{(\bullet)}$
}

\author{
L.A. Pol ${ }^{(*)}$, J. Asensio $^{(*)}$ y J.A. Pero-Sanz ${ }^{(* *)}$ \\ Resumen Se ha realizado una serie de ensayos de compresión plana en caliente en muestras de un acero \\ microaleado con niobio y titanio en una máquina Servotest, en probetas trapezoidales de \\ aproximadamente $10 \mathrm{~mm}$ de espesor. Se efectuaron dos series de ensayos con dos velocidades de \\ deformación, 5 y $15 \mathrm{~s}^{-1}$, y temperaturas de deformación entre 850 y $1.025^{\circ} \mathrm{C}$. Los datos \\ experimentales fueron corregidos por rozamiento probeta-herramientas, ausencia de condiciones de \\ deformación plana y aumento de la temperatura en la muestra debido a que la deformación no es \\ estrictamente adiabática, para obtener las curvas isotermas equivalentes en tracción. Dichas curvas \\ han sido modelizadas según dos tipos de ecuaciones constitutivas que caracterizan el proceso de \\ ablandamiento que tiene lugar concurrente a la deformación. Se calculan los coeficientes de las \\ mismas por desarrollo en serie de Taylor alrededor de un punto inicial próximo a la solución y \\ posteriormente, por mínimos cuadrados, se compara con los valores experimentales para obtener un \\ valor optimizado de dichos coeficientes, iterando hasta la convergencia.
}

Palabras clave: Modelización. Deformación en caliente. Aceros microaleados.

\section{High temperature stress-strain curve fitting of a $\mathrm{Nb}-\mathrm{Ti}$ microalloyed steel}

\begin{abstract}
A series of hot plane strain compression tests have been conducted on a $\mathrm{Nb}$-Ti microalloyed steel in a Servotest machine on samples of approx. $10 \mathrm{~mm}$ in thickness. Two sets of tests at strain rates of $5 \mathrm{~s}^{-1}$ and $15 \mathrm{~s}^{-1}$ were used and monitored the stress-strain behavior at deformation temperatures varying from 850 to $1,025^{\circ} \mathrm{C}$. The experimental data were corrected for friction, deviation from plane strain compression (PSC) conditions and adiabatic rise of temperature, to give the isothermal equivalent tensile stress-strain curves. Thereafter these curves have been modeled by two types of equations depending on the softening mechanism operating during the deformation process and calculated the value of the coefficients in the equation by the application of a Taylor series around an initial point close to the solution. Finally, by the application of the least squared method, the previous values are compared to the experimental ones in order to determine an optimized value of the coefficients in the equations whch model the stress-strain behaviour of the steel.
\end{abstract}

Keywords: Modeling. Hot deformation. Microalloyed steels.

\section{INTRODUCCIÓN}

(•) Trabajo recibido el día 1 de marzo de 1996.

(*) Dpto. de Ciencia de los Materiales e Ingeniería Metalúrgica. E.T.S.I. de Minas. Universidad de Oviedo. Independencia, 13. 33004-Oviedo (España).

(**) Dpto. de Ingeniería de Materiales. Laboratorio de Metalotecnia. E.T.S.I. de Minas. Universidad Politécnica de Madrid. Ríos Rosas, 21. 28003-Madrid (España).
El presente trabajo se centra en el ajuste de los puntos que configuran las curvas tensión-deformación experimentales de un acero microaleado con niobio y titanio. Dicho ajuste tiene gran interés en la industria de la conformación en caliente de metales y aleaciones, pues permite determinar valores de 
las constantes en los modelos de comportamiento y así conseguir un mayor rendimiento en la práctica operativa, mayores porcentajes de éxito en las especificaciones relativas al producto, mejorar las propiedades mecánicas de los productos (límite elástico, tenacidad, etc.) y, en general, suministrar al consumidor un producto de mayor valor añadido.

La modelización de los procesos de conformado en caliente se refiere al comportamiento mecánico, a la evolución microestructural y a su interdependencia, y en la actualidad es una herramienta esencial de la fabricación que está permitiendo conocer con más precisión las relaciones funcionales entre los diversos parámetros metalúrgicos y el comportamiento en deformación. Su objetivo final consiste en la optimización de los procesos de fabricación para obtener productos que, en servicio, tengan propiedades mejoradas.

En lo referente a los modelos de laminación industrial, todos actúan bajo el denominador común de abundante información referente a datos básicos relativos a las respuestas del material a las diferentes variables de proceso, agrupados en bases de datos que se clasifican según líneas de producto. Habitualmente, se engloban en tres categorías:

- La tensión de fluencia, que es el valor de entrada para la determinación de magnitudes básicas, tales como esfuerzo y potencia. Paralelamente, es un dato de partida en la modelización dinámica por elementos finitos de las evoluciones másicas en el curso de la deformación.

- Las cinéticas y magnitudes de cambios microestructurales, que a su vez influyen en la tensión de fluencia durante las pasadas de laminación, y que controlan las propiedades del producto.

- El estado superficial, que se puede expresar en función de la transferencia de calor y de los coeficientes de rozamiento, aunque en la práctica viene condicionada, fundamentalmente, por el comportamiento de la capa de óxido superficial.

Para la descripción de las curvas tensión-deformación se han adoptado las expresiones generales relativas a las ecuaciones constitutivas disponibles en modelos que, referidos en la literatura técnica, mejor describen el comportamiento de los aceros microaleados a alta temperatura. La aplicación de un ajuste matemático por mínimos cuadrados a un conjunto de datos experimentales $\sigma-\epsilon$, tales como los suministrados por máquinas de compresión plana, permite determinar la mejor de las estimas de las curvas tensión-deformación a la temperatura y velocidad de deformación del ensayo, por medio de un método de cálculo iterativo que se describe en el capítulo siguiente.

\section{DATOS EXPERIMENTALES Y CONDICIONES DE ENSAYO}

Se han efectuado ensayos de compresión plana en una acero microaleado cuya composición se describe en la tabla I.

Dicha composición se obtuvo por fusión en un horno de vacío a partir de ferroalaeciones y chatarra de alta pureza, al objeto de evitar la oxidación del titanio. Los contenidos de elementos residuales se limitaron a $0,014 \%$ P y $0,001 \% \mathrm{~S}$, para obtener un acero de gran pureza próximo a las mejores calidades obtenidas en los procesos de la siderurgia integral (oxiconvertidor y metalurgia secundaria). Durante el proceso de fusión, se adicionó granalla de aluminio para desoxidar el baño, previamente a la adición de ferrotitanio, y en cierto momento se presurizó el horno con nitrógeno con objeto de controlar la proporción Ti:N en el baño. El caldo solidifica hasta temperatura ambiente en forma de lingote de sección circular. Posteriormente, se despuntó y se extruyó en caliente a $1.200{ }^{\circ} \mathrm{C}$ a través de la embocadura de una matriz de sección rectangular de dimensiones próximas a las de las probetas a ensayar. La zona correspondiente al "metal muerto" fue desechada, y la barra cortada en trozos iguales según su longitud. Se obtuvieron paralelepípedos rectos que tras mecanizado y rectificado, tuvieron por dimensiones $50 \times 30 \times 10 \mathrm{~mm}$ (largo $\times$ ancho $\times$ espesor). Posteriormente, se recubrieron electrolíticamente con cromo para dar una capa de aproximadamente $30 \mu \mathrm{m}$ para evitar la oxidación superficial de las mismas durante su posterior tratamiento termomecánico. Todos los ensayos consistieron en un recalentamiento en horno de resistencias radiantes hasta una temperatura de empapado de $1.200{ }^{\circ} \mathrm{C}$ durante $20 \mathrm{~min}$. Por término medio se tardaron 5 min. en alcanzar dicha temperatura. Cada muestra se enfrió posteriormente al aire hasta unos $20{ }^{\circ} \mathrm{C}$ por encima de la temperatura de deformación, momento en el cual se introdujo en el horno de deformación, en el que dicha muestra equilibra su temperatura, para inmediatamente después proceder a su deformación. La evolución de la temperatura de la muestra se registró en continuo mediante un termopar en el centro del espesor de la probeta durante toda la historia térmica, y sus datos fueron almacenados con los de deformación.

TABLA I.- Composición química básica del acero objeto de estudio, \% en peso

TABLE I.- Basic chemical composition of the studied steel, \% in wt.

\begin{tabular}{|c|c|c|c|c|c|c|}
\hline $\mathrm{C}$ & $\mathrm{Mn}$ & $\mathrm{Al}$ & $\mathrm{Si}$ & $\mathrm{Nb}$ & $\mathrm{Ti}$ & $\mathrm{N}, \mathrm{ppm}$ \\
\hline 0,064 & 1,46 & 0,023 & 0,02 & 0,064 & 0,062 & 60 \\
\hline
\end{tabular}


Los ensayos de deformación se efectuaron a velocidad y temperatura de deformación constantes, siendo dos las velocidades de deformación ensayadas, 5 y $15 \mathrm{~s}^{-1}$, y temperaturas de deformación en el intervalo de 850 a $1.050{ }^{\circ} \mathrm{C}$. Todos los ensayos se llevaron a cabo en una máquina de compresión plana Servotest, del Laboratorio de Deformación en Caliente, en el Departamento de Ingeniería de Materiales de la Universidad de Sheffield (R.U.). Los datos suministrados por la máquina se almacenaron en un soporte informático para su posterior análisis. Las curvas obtenidas tras dicho análisis son las de tensión-deformación equivalentes en tracción y corregidas por ausencia de condiciones adiabáticas durante su deformación, y se corresponden con la colección de datos experimentales sobre los que se ajustan los modelos antes mencionados.

\section{ANÁLISIS Y DISCUSIÓN DE RESULTADOS}

\subsection{Descripción de las ecuaciones de modelización}

Durante los procesos de deformación en caliente se han asumido tradicionalmente dos tipos de comportamientos en atención a los cambios microestructurales que actúan como mecanismos de ablandamiento concurrentes con la misma. Cada uno de ellos se corresponde con un dominio en la curva $\sigma$ $\epsilon$. Dichos ámbitos surgen del análisis relativo de los distintos comportamientos distinguibles en metales y sus aleaciones, cuando se deforman en caliente.

En primer lugar, se encuentran aquellos materiales cuyo mecanismo de ablandamiento más importante es la restauración dinámica a altas temperaturas. En éstos, la tensión de fluencia aumenta rápidamente con la deformación aplicada, hasta un punto en el que el material se satura y no se obtiene aumento del nivel de tensión aun cuando aumente la deformación aplicada. Este proceso de ablandamiento está muy condicionado por la temperatura y por la velocidad de deformación, y es típico del Fe$\alpha$, del aluminio y sus aleaciones, etc. La ecuación constitutiva del mismo fue descrita por Sah y col. en 1969 y viene dada por la siguiente expresión:

$$
\sigma=\sigma_{0}+\left(\sigma_{S S}-\sigma_{0}\right)\left(1-\exp \left(\frac{-\varepsilon}{\varepsilon_{r}}\right)\right)^{n}
$$

Donde:

$\sigma_{0}=$ Tensión aplicada al comienzo de la deformación plástica

$\sigma_{\mathrm{ss}}=$ Valor de la tensión en el estado estacionario (también llamada tensión de saturación). $\epsilon_{\mathrm{r}}=$ Deformación de relajación, que es el valor de la deformación requerido para alcanzar un valor del endurecimiento por deformación prefijado.

$n=$ Exponente de endurecimiento por deformación.

En esta ecuación, el término exponencial determina la velocidad a la que se alcanza el estado estacionario. En el presente estudio se ha adoptado:

$$
\frac{1}{\varepsilon_{\mathrm{r}}}=\mathrm{C}
$$

El segundo tipo de materiales engloba a los aceros austeníticos, aleaciones de cobre, aleaciones de níquel, etc. Estos materiales verifican ablandamientos por recristalización dinámica cuando se alcanza en el material un valor crítico de la deformación $\epsilon_{\mathrm{c}}$ correspondiente a una fracción $x$ de la deformación de pico (o deformación correspondiente a la tensión máxima) $\epsilon_{\mathrm{p}}$ (Fig. 1):

$$
\varepsilon_{\mathrm{c}}=X \cdot \varepsilon_{\mathrm{p}}
$$

Leduc describió en 1980 el ablandamiento por recristalización dinámica como:

$$
\Delta \sigma=\left(\sigma_{\mathrm{ss}}(e)-\sigma_{\mathrm{ss}}\right) \times\left(1-\exp \left(-\mathrm{k} \frac{\left(\varepsilon-\mathrm{x} \varepsilon_{\mathrm{p}}\right)}{\varepsilon_{\mathrm{p}}}\right)\right)^{\mathrm{m}}
$$

siendo la ecuación constitutiva global el resultado de:

$$
\sigma=\sigma_{\mathrm{ss}}(e)-\Delta \sigma
$$

Los parámetros de las últimas ecuaciones se reflejan en el gráfico de la figura 1, e indican:

$\sigma_{\mathrm{ss}}(e)=$ Tensión del estado estacionario bajo la hipótesis de ablandamiento sólo por procesos de restauración dinámica.

$\epsilon_{\mathrm{p}}=$ Deformación correspondiente a la tensión de pico.

$\sigma_{\mathrm{ss}}=$ Tensión del estado estacionario resultante del equilibrio entre el endurecimiento por deformación y el ablandamiento por restauración y recristalización dinámicas.

\subsection{Cálculo de los coeficientes de las ecuaciones}

En el presente trabajo se ha asumido la validez de las ecuaciones descritas en el subcapítulo anterior y se ha procedido al cálculo de los parámetros que mejor se ajustan a los datos experimentales. 
En el análisis de los datos experimentales, y en atención a la morfología de las curvas, se han estudiado de modo separado los dos comportamientos previamente descritos:

- Estudio de la porción de datos que en la curva se corresponde con los fenómenos de endurecimiento por deformación y restauración dinámica únicamente (tramo T1 en la Fig. 1).

- Porción de la curva indicada como T2 en la curva de la figura 1, en la que tiene lugar el ablandamiento por recristalización dinámica.

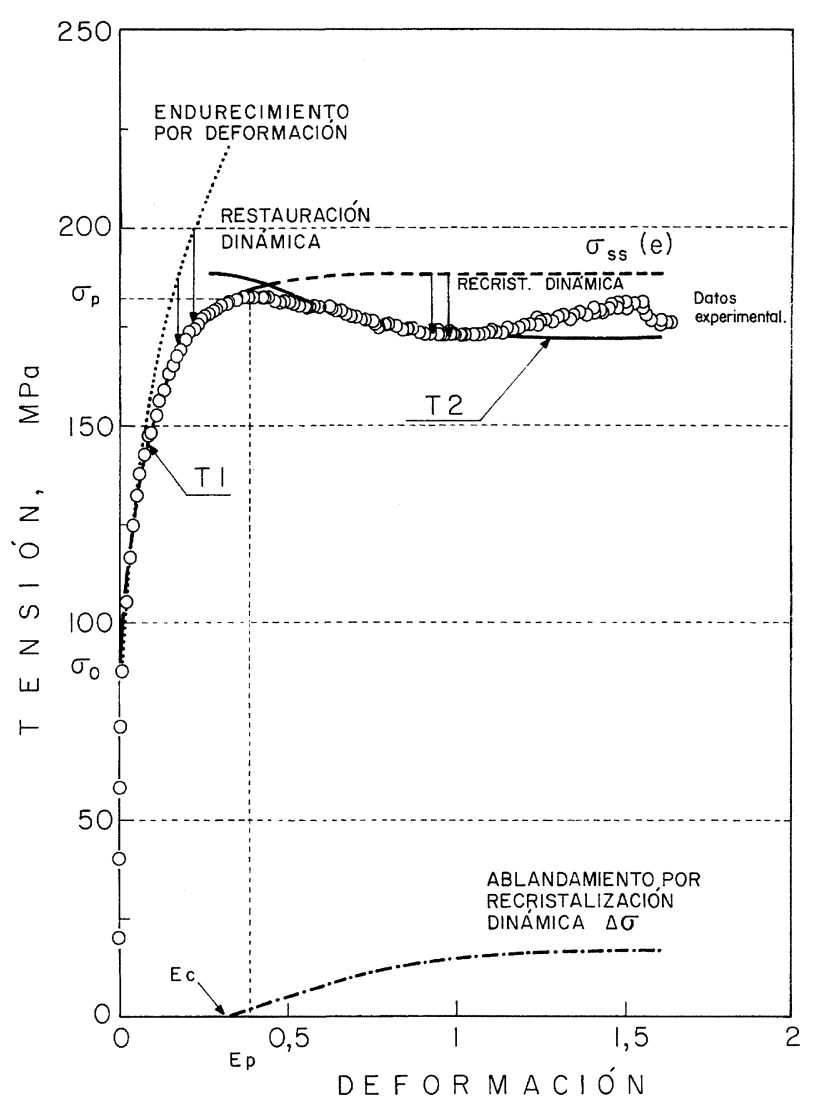

FIG. 1.- Datos experimentales corregidos por el rozamiento, por la ausencia de condiciones de deformación plana ideal y por el aumento de la temperatura debido a ensayos en condiciones de deformación cuasi-adiabáticas. Se muestran las regiones T1 y T2 y se señalan los mecanismos de ablandamiento que tienen lugar así como los valores críticos de la tensión y de la deformación.

FIG. 1.- Experimental data corrected for friction, absence of pure plane strain conditions and for rise in the temperature during the test due to existence of quasi-adiabatic deformation conditions. The regions $\mathrm{T} 1$ and $\mathrm{T} 2$ are shown, with indication of the softening mechanism taking place together with the critical values of the stress and strain.
Para la porción de datos experimentales en la región T1, se empleará la ec. [1] que, con las consideraciones efectuadas, quedaría como:

$$
\sigma=\sigma_{0}+\left(\sigma_{\mathrm{ss}}-\sigma_{0}\right)(1-\exp (-C \varepsilon))^{\mathrm{n}}
$$

Para el cálculo de los coeficientes $\sigma_{0}, \sigma_{\mathrm{ss}}, C$ y $n$, que permitan ajustar una curva teórica a la secuencia de puntos obtenida experimentalmente, se aplicó el modelo matemático que se describe en el apartado siguiente.

Para el tramo T2, las ecuaciones de modelización serán [4] y [5], siendo los parámetros cuyo valor se trata de determinar (incógnitas): $\sigma_{\mathrm{ss}}, k, x, \epsilon_{\mathrm{p}}$ y $m$, por aplicación de un modelo matemático similar al empleado en el tramo anterior.

\subsubsection{Modelo matemático para las curvas en el tramo $T 1$}

Para determinar coeficientes que proporcionen un ajuste óptimo de los datos experimentales, se desarrolló en serie cada ecuación del tramo en cuestión y, posteriormente, se comparó con el valor experimental por el método de mínimos cuadrados. Se obtiene, así, un valor optimizado para dicho coeficiente.

En la ecuación del tramo T1, el desarrollo en serie de Taylor alrededor de un punto inicial próximo a la solución se expresa como:

$$
\begin{aligned}
& \sigma_{\text {Taylor }}=(\sigma)_{0}+\left(\frac{\partial \sigma}{\partial \sigma_{0}}\right)_{0}\left(\sigma_{0}-\sigma_{0}^{\prime}\right)+\left(\frac{\partial \sigma}{\partial \sigma_{\mathrm{ss}}}\right)_{0} \\
& \left(\sigma_{\mathrm{ss}}-\sigma_{\mathrm{ss}}^{\prime}\right)+\left(\frac{\partial \sigma}{\partial C}\right)_{0}\left(C-C^{\prime}\right)+\left(\frac{\partial \sigma}{\partial n}\right)_{0}\left(n-n^{\prime}\right)
\end{aligned}
$$

donde los coeficientes investigados son $\sigma_{0}, \sigma_{\mathrm{ss}}, C \mathrm{y}$ $n$, que se consideran como variables.

En esta ecuación, $(\sigma)_{0}$ es la ecuación constitutiva particularizada para el punto de comienzo del proceso iterativo $\left(\sigma_{0}^{\prime}, \sigma^{\prime}{ }_{s s}, C^{\prime}\right.$ y $n$ '). Además:

$$
\left(\frac{\partial \sigma}{\partial \sigma_{0}}\right)_{0},\left(\frac{\partial \sigma}{\partial \sigma_{\mathrm{ss}}}\right)_{0},\left(\frac{\partial \sigma}{\partial C}\right)_{0},\left(\frac{\partial \sigma}{\partial n}\right)_{0}
$$

son las derivadas parciales de la ecuación constitutiva con respecto a los coeficientes investigados, también particularizados para el punto de partida del proceso iterativo, que es el que se toma como solución inicial de los cuatro parámetros indicados.

El desarrollo de la expresión correspondiente a la ec. [7] se puede simplificar notablemente 
adoptando un valor fijo para el exponente $n$. En el presente caso se ha adoptado el valor 0,59 , que permite alcanzar la convergencia del proceso iterativo y llegar a una expresión del tipo:

$$
\begin{gathered}
\sigma_{\text {Taylor }}=(\sigma)_{0}+\left(\frac{\partial \sigma}{\partial \sigma_{0}}\right)_{0}\left(\sigma_{0}-\sigma_{0}^{\prime}\right)+\left(\frac{\partial \sigma}{\partial \sigma_{s s}}\right)_{0} \\
\left(\sigma_{s s}-\sigma_{s s}^{\prime}\right)+\left(\frac{\partial \sigma}{\partial C}\right)_{0}\left(C-C^{\prime}\right)
\end{gathered}
$$

con un nuevo valor de $(\sigma)_{0}$ dado por:

$$
(\sigma)_{0}=\left[\sigma_{0}+\left(\sigma_{\mathrm{ss}}-\sigma_{0}\right)(1-\exp (-C \varepsilon))^{\mathrm{n}}\right]_{0}
$$

El valor de las derivadas parciales:

$$
\begin{gathered}
\left(\frac{\partial \sigma}{\partial \sigma_{0}}\right)_{0}=\left[1-(1-\exp (-C \varepsilon))^{\mathrm{n}}\right]_{0} \\
\left(\frac{\partial \sigma}{\partial \sigma_{\mathrm{ss}}}\right)_{0}=\left[(1-\exp (-C \varepsilon))^{\mathrm{n}}\right]_{0} \\
\left(\frac{\partial \sigma}{\partial C}\right)_{0}=\left[\left(\sigma_{\mathrm{ss}}-\sigma_{0}\right) n(1-\exp (-C \varepsilon))^{\mathrm{n}-1}\right. \\
(-\exp (-C \varepsilon))(-\varepsilon)]_{0}
\end{gathered}
$$

Todas ellas han de calcularse para la solución inicial:

$$
\sigma_{0}=\sigma_{0}^{\prime} \sigma_{\mathrm{ss}}=\sigma_{\mathrm{ss}}^{\prime} C=C y n=n^{\prime}
$$

Los datos que proporciona $\sigma_{\text {Taylor }}$ obtenida de esta manera, se comparan con los valores experimentales $\sigma_{\text {exp }}$ empleando el método de mínimos cuadrados a fin de obtener un valor optimizado de los coeficientes:

$$
\Omega_{1}=\sum_{\mathrm{i}}^{\mathrm{N}}\left(\sigma_{\exp }(\mathrm{i})-\sigma_{\text {Taylor }}\right)^{2}
$$

donde el subíndice $i$ corresponde a pares de valores experimentales $\left(\sigma_{\mathrm{i}}, \epsilon_{\mathrm{i}}\right)$.

La condición de mínimos cuadrados implica que el sumatorio $\Omega_{1}$ sea mínimo, y para ello es necesario que las derivadas parciales con respecto a los coeficientes investigados $\sigma_{0}, \sigma_{\mathrm{ss}}$ y $C$ han de ser iguales a cero:

$$
\left(\frac{\partial \Omega_{1}}{\partial \sigma_{0}}\right)=0
$$

$$
\left(\frac{\partial \Omega_{1}}{\partial \sigma_{\mathrm{ss}}}\right)=0
$$

$$
\left(\frac{\partial \Omega_{1}}{\partial C}\right)=0
$$

Las ecs. [14-16] corresponden a un sistema de tres ecuaciones y tres incógnitas: $\sigma_{0}, \sigma_{\mathrm{ss}}$ y $C$. Una vez resuelto, la solución obtenida sirve como nuevo punto inicial mejorado para volver a recalcular los coeficientes. El proceso iterativo se considera concluido cuando se alcanza una convergencia prefijada en la solución.

\subsubsection{Efecto de los coeficientes en el modelo. Constante de endurecimiento por deformación $n$ y constante $C$}

Como se ha indicado anteriormente, en el proceso de cálculo no se contempla un estudio del óptimo de $n$ (exponente en la ec. [1]), al observarse que el proceso de cálculo no converge en torno a ninguna solución.

Por tanto, se ha adoptado un valor inicial de $n$ que permita el ajuste óptimo de la curva a los datos experimentales. Cuanto más pequeño sea el valor de $n$, más rápido resulta el incremento en los niveles de tensión, tal y como se indica en la figura 2. Sin embargo, la búsqueda de valores mejorados del coeficiente $n$, no parece estar justificada dado que el coeficiente $C$, incógnita en el proceso de cálculo, tiene un efecto semejante al del coeficiente $n$ en el ajuste de los datos experimentales a la curva de modelización teórica, pero actuando de forma opuesta. (Fig. 3).

Por tanto, resulta satisfactorio en el proceso de ajuste centrarse en el valor óptimo del coeficiente $C$ cada vez que se adopta un valor de $n$ de la forma indicada previamente.

\subsubsection{Curvas en el tramo $T 2$}

El modo práctico en que se deducen los valores numéricos de los coeficientes es similar al indicado en la sección previa. Únicamente se apunta la secuencia práctica a seguir para ajustar los valores experimentales.

Una vez ajustados los datos experimentales del primer tramo y derivada la ecuación que modeliza su tendencia, se determina un valor para $\sigma_{\text {ss }}$ que en este apartado corresponde al valor extrapolado $\sigma_{\mathrm{ss}}(e)$ (Fig. 1).

Al calcularse la diferencia: 


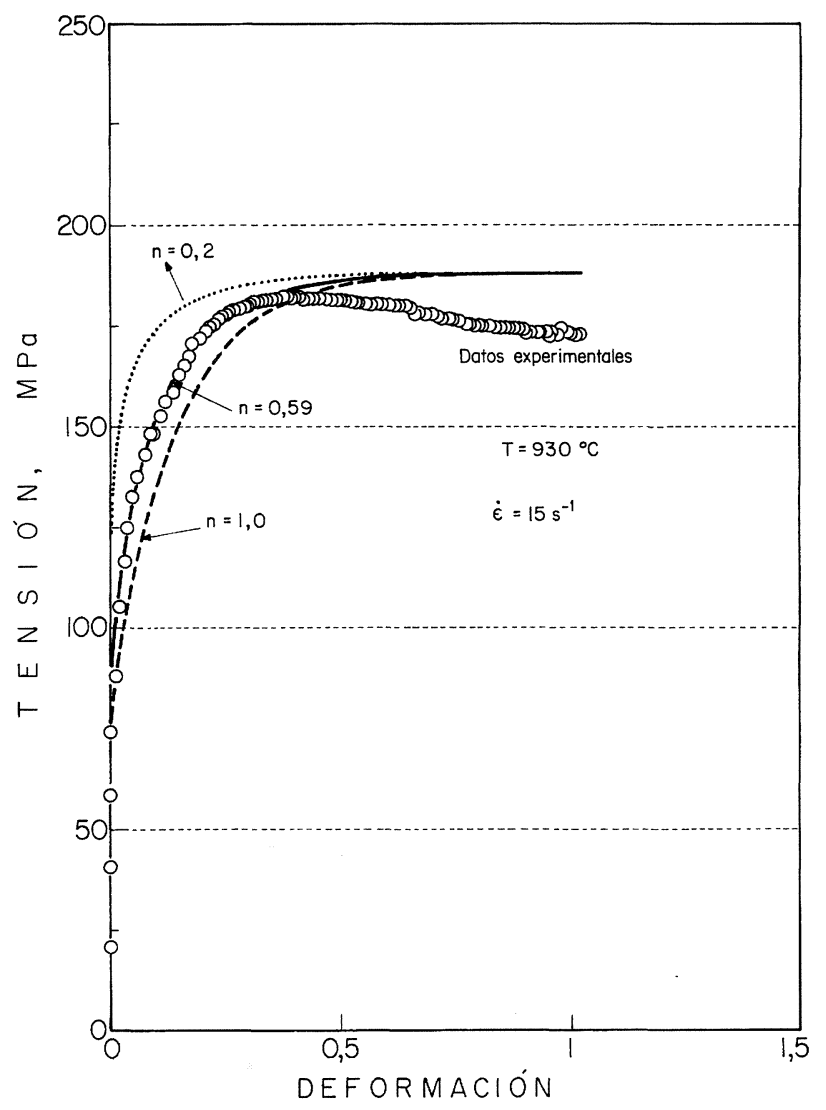

FIG. 2.- Ajuste de los datos experimentales para diferentes valores del coeficiente $n$ de endurecimiento por deformación a $930{ }^{\circ} \mathrm{C}$ y $15 \mathrm{~s}^{-1}$ en la región $\mathrm{T} 1(C=7,069)$.

FIG. 2.- Fitting of the experimental data for different values of the strain hardening coefficient $\mathrm{n}$. The deformation characteristics are $930^{\circ} \mathrm{C}$ and $15 \mathrm{~s}^{-1}$.

$$
\Delta \sigma_{\exp }=\sigma_{\text {ss }}(e)-\sigma_{\exp }
$$

se obtiene una colección de puntos que se procesan como colección de datos experimentales a los que se ajusta la ec. [4]. A continuación, se sigue paso a paso un proceso similar al indicado previamente:

- Desarrollo en serie de Taylor de la ec. [4] alrededor del punto inicial de coordenadas $\left(\sigma^{\prime}{ }_{s s}, k\right.$, m' y $\epsilon^{\prime}$ ):

$$
\begin{aligned}
& \Delta \sigma_{\text {Taylor }}=(\Delta \sigma)_{0}+\left(\frac{\partial \Delta \sigma}{\partial \sigma_{\mathrm{ss}}}\right)\left(\sigma_{\mathrm{ss}}-\sigma_{\mathrm{ss}}^{\prime}\right)+ \\
& +\left(\frac{\partial \Delta \sigma}{\partial k}\right)\left(k-k^{\prime}\right)+\left(\frac{\partial \Delta \sigma}{\partial x}\right)\left(x-x^{\prime}\right)
\end{aligned}
$$

- Se plantea la condición de mínimos cuadrados por comparación de los valores experimentales con la aproximación dada por Taylor:

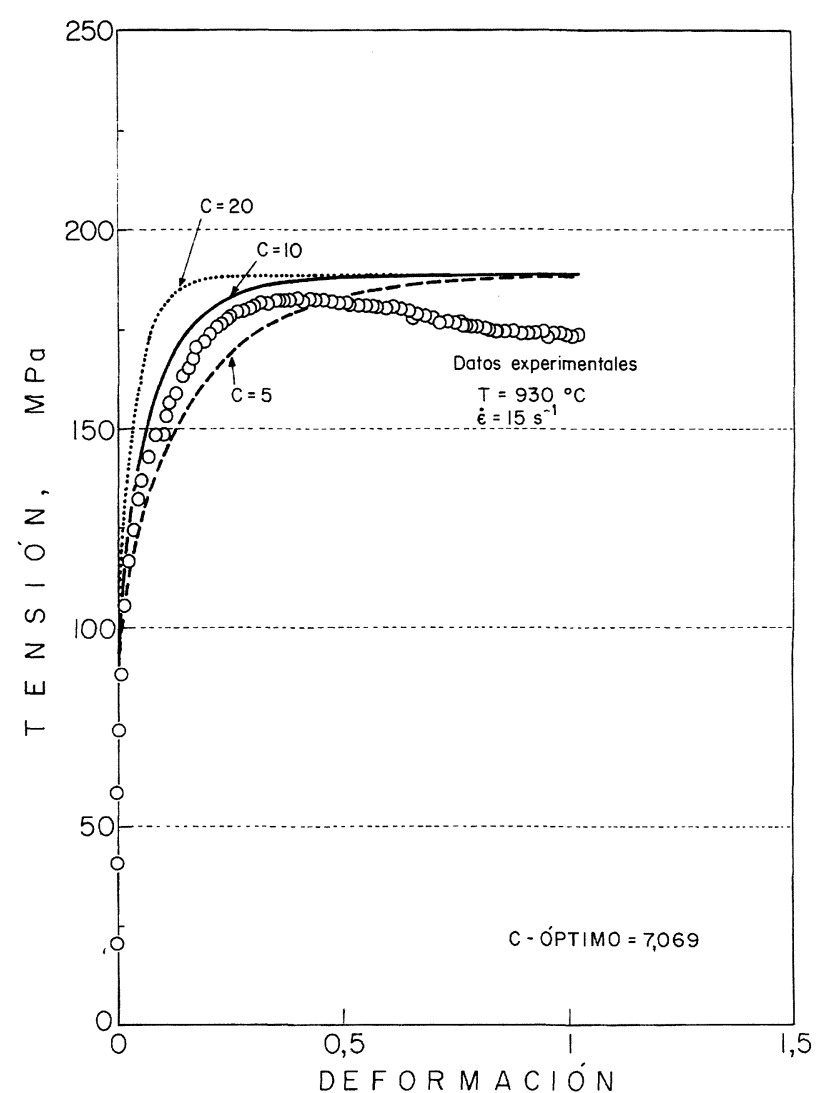

FIG. 3.- Ajuste de los datos experimentales en el tramo T1 a $930{ }^{\circ} \mathrm{C}$ y $15 \mathrm{~s}^{-1}$ con indicación del óptimo valor de $C(n=0,59)$. Se aprecia como un aumento de su valor provoca que se alcance antes el nivel de saturación de tensión.

FIG. 3.- Experimental data fitting in the region $\mathrm{T} 1$ at $930{ }^{\circ} \mathrm{C}$ and $15 \mathrm{~s}^{-1}$, with indication of the optimum value of $\mathrm{C}(\mathrm{n}=0,59)$. An increase in $\mathrm{K}$ that contributes to an early reach of the stress saturation level is observed.

$$
\Omega_{2}=\sum_{\mathrm{i}}^{\mathrm{N}}\left(\Delta \sigma_{\mathrm{exp}}-\Delta \sigma_{\text {Taylor }}\right)^{2}
$$

- Nuevamente se ha de conseguir que el sumatorio $\Omega_{2}$ sea mínimo, para ello, las derivadas parciales con respecto a los coeficientes investigados $\sigma_{\mathrm{ss}}$, $k$ y $x$ han de ser iguales a cero:

$$
\begin{aligned}
& \left(\frac{\partial \Omega_{2}}{\partial \sigma_{\mathrm{ss}}}\right)=0 \\
& \left(\frac{\partial \Omega_{2}}{\partial k}\right)=0 \\
& \left(\frac{\partial \Omega_{2}}{\partial x}\right)=0
\end{aligned}
$$


Se tiene un sistema de tres ecuaciones con tres incógnitas, $\sigma_{\mathrm{ss}}, k, \mathrm{y} x$, que se resuelve, y su solución constituye un valor mejorado de la solución inicial que se toma como punto de comienzo del proceso iterativo al volver a retomar el paso [1].

- El proceso iterativo se considera concluido cuando se alcanza un nivel de convergencia satisfactorio.

\subsubsection{Efecto de los coeficientes en el modelo. Coeficiente $m$}

En este caso, el valor se estimó de modo similar al del coeficiente $n$ del caso anterior. Cada vez que se adopta un valor para dicho coeficiente (Fig. 4), el resto de los coeficientes corrige el posible error en que se haya podido incurrir en dicha selección.

Deformación de pico: $\epsilon_{\mathrm{p}}$

Con respecto a la deformación de pico, hay que proceder a estimar su valor en cada una de las cur-

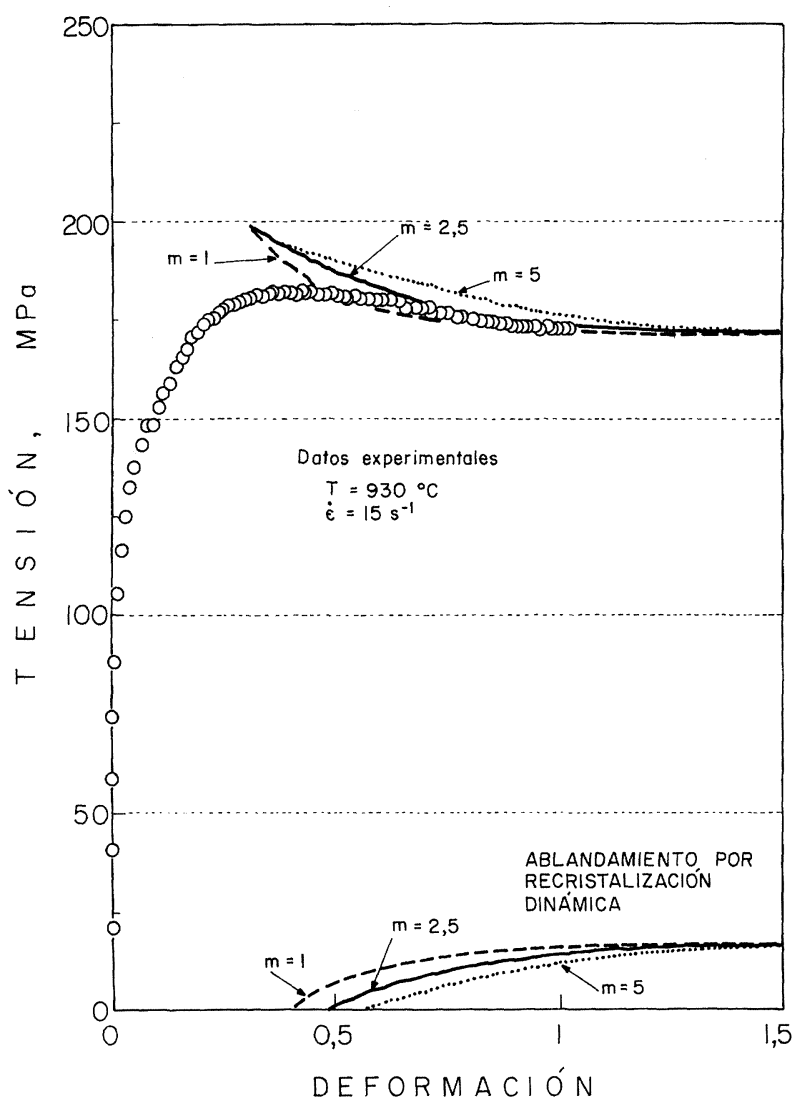

FIG. 4.- Efecto de los valores asignados al coeficiente $m$ en la ecuación que modeliza la recristalización dinámica (región T2) en la bondad de ajuste a los datos experimentales $\left(930^{\circ} \mathrm{C}, 15 \mathrm{~s}^{-1}\right)$.

FIG. 4.- Effect of the values assigned to the coefficient $\mathrm{m}$ in the equation which models the dynamic recrystallization (region $\mathrm{T} 2$ ) showing the best fit to the experimental data $\left(930^{\circ} \mathrm{C}, 15 \mathrm{~s}^{-1}\right)$. vas experimentales y mantener fijo su valor en el resto del proceso de cálculo.

\section{Coeficientes $X$ y $k$}

También se ha investigado el efecto de los coeficientes $X$ y $k$ en el ajuste de la curva a los datos experimentales, tal y como se indica en las figuras 5 y 6.

\subsection{Criterios racionales de selección}

En este proceso matemático, todos los coeficientes investigados en las ecuaciones proceden del sumatorio particularizado para todos los datos experimentales en cada ensayo en concreto. Sin embargo, la elección de los puntos inicial y final que afectan al sumatorio parece constituir una decisión crítica que afecta a la solución del problema; esto es, al valor de los coeficientes investigados y, en último extremo, al valor de la tensión cuyo objetivo último es el que se persigue. Un criterio

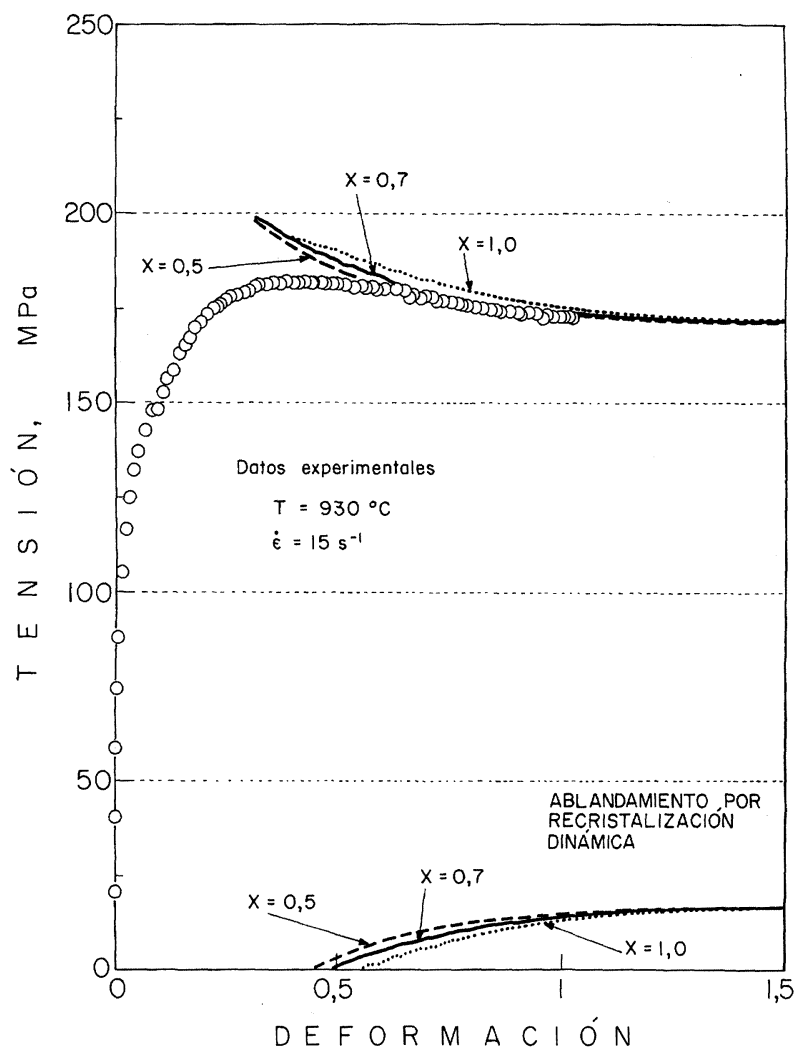

FIG. 5.- Efecto del coeficiente $x$ en las características de ablandamiento del acero y ajuste de la ecuación modelizada a los datos experimentales $\left(930^{\circ} \mathrm{C}, 15 \mathrm{~s}^{-1}\right)$.

FIG. 5.- Effect of the coefficient $\mathrm{x}$ in the characteristics of softening in the steel and modelled equation adjusted to the experimental data $\left(930^{\circ} \mathrm{C}, 15 \mathrm{~s}^{-1}\right)$. 


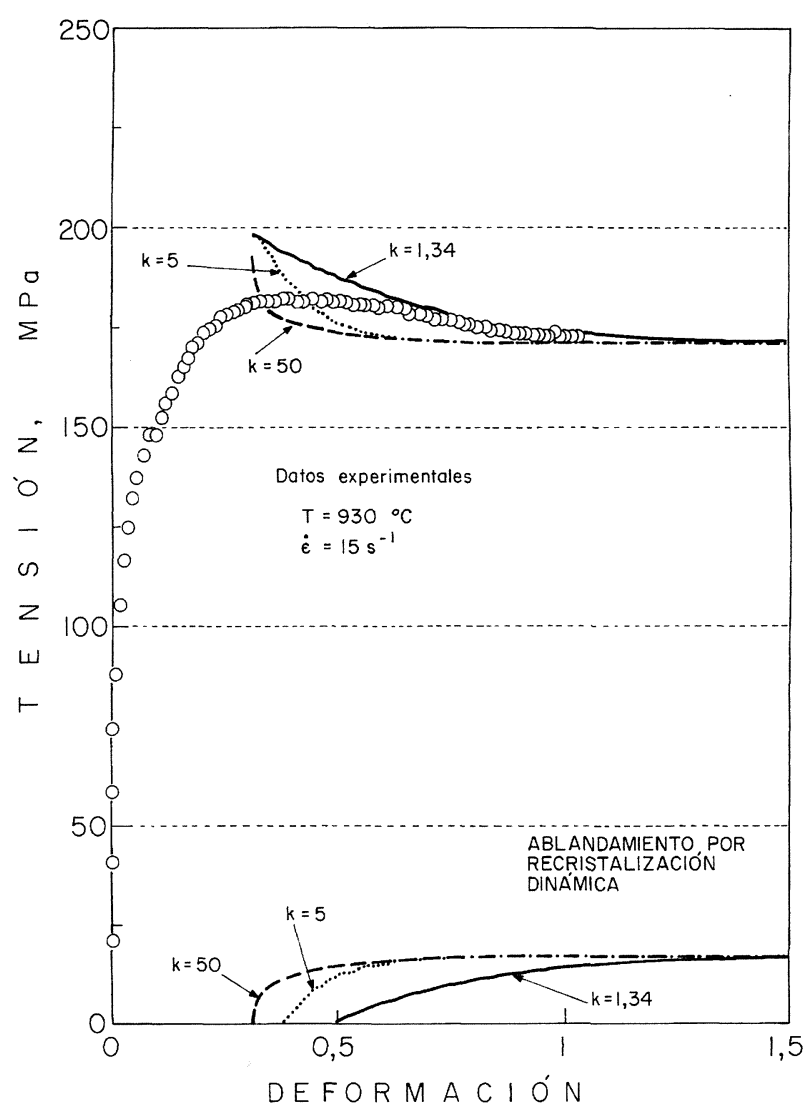

Fig. 6.- Efecto de la constante $k$ en el ajuste a los datos experimentales a $930{ }^{\circ} \mathrm{C}$ y $15 \mathrm{~s}^{-1}$ en la región $\mathrm{T} 2$.

FIG. 6.- Effect of the constant $\mathrm{K}$ in the adjustment of the experimental data at $930^{\circ}$ and $15 \mathrm{~s}^{-1}$ in the region $\mathrm{T} 2$.

racional de selección puede ser el que se propone a continuación:

\section{Para la región $\mathrm{T} 1$}

El sumatorio comienza a partir del punto experimental 20 y finaliza en el máximo de la tensión. El punto de comienzo viene marcado por un valor mínimo de la deformación que puede tomarse igual a 0,05 , dado que para valores menores de ésta, la colección de valores experimentales acusa grandes oscilaciones motivadas por las propias oscilaciones de la máquina.

\section{Para la región $\mathrm{T} 2$}

Los sumatorios se han de extender desde el punto final en la sección T1 hasta el último valor del estado estacionario, que en el presente cálculo se adopta como aquella colección de puntos que manteniendo un valor constante de tensión suceden a la tensión de pico. Lógicamente, no han de coincidir con los últimos datos experimentales registra- dos, dado que en algunos ensayos, los datos corregidos por rozamiento adherente y ausencia de condiciones isotermas muestran valores anómalos respecto al modelo.

Dicha tendencia también se observa en otros resultados experimentales de curvas deformadas a $5 \mathrm{~s}^{-1}$ (Fig. 7a); entre éstas, el fenómeno señalado de aumento en el nivel de tensión, es más acusado a mayor temperatura -curva de $1.025{ }^{\circ} \mathrm{C}$-. Este comportamiento experimental distinto de las condiciones teóricas puede deberse a un aumento del nivel de esfuerzos, quizá debido al endurecimiento que pudiese provocar la dilatación térmica de la "parte fría" de la herramienta que entra en el horno de ensayo en el momento de verificarse la deformación. Esta tendencia también es observable en las curvas a $15 \mathrm{~s}^{-1}$ (Fig. 7b) con un efecto más marcado en las muestras ensayadas a baja temperatura.

\section{CONCLUSIONES}

Como resultado de la técnica de modelización propuesta resulta posible obtener las curvas tensión-deformación verdaderas equivalentes en tracción ( $T=$ cte.) de un acero microaleado con niobio y titanio. La técnica es aplicable a cualquier otro tipo de acero microaleado o no, así como a materiales que sólo verifiquen ablandamientos por restauración dinámica (aleaciones de aluminio, etc.) en cuyo caso el ajuste resultaría más sencillo.

Para el acero en cuestión, a partir de la colección de datos experimentales a distintas temperaturas y velocidades de deformación, se han observado dos regiones, atendiendo a los mecanismos de ablandamiento que actúan concurrentes a la deformación: restauración dinámica y recristalización dinámica. Cada colección de datos experimentales se puede modelizar por medio de dos ecuaciones constitutivas diferentes. Dichas ecuaciones se simbolizan por dos curvas, una para cada uno de los tramos referidos (T1 y T2).

El método empleado consiste en la aplicación de las ecuaciones constitutivas comúnmente aceptadas en la literatura, de las que se procede a realizar un desarrollo en serie de Taylor para cada tramo. El cálculo de los coeficientes de las ecuaciones se simplifica al desarrollar en serie sólo hasta la primera derivada cada una de estas particularizada para una "solución inicial". Ello permite comparar la colección de puntos experimentales con los valores deducidos por Taylor mediante mínimos cuadrados y deducir los nuevos valores de los coeficientes de la ecuación. Se plantea un sistema de ecuaciones a partir de la condición impuesta consistente en minimizar las diferencias al cuadrado entre los datos experimentales y el valor de Taylor. 

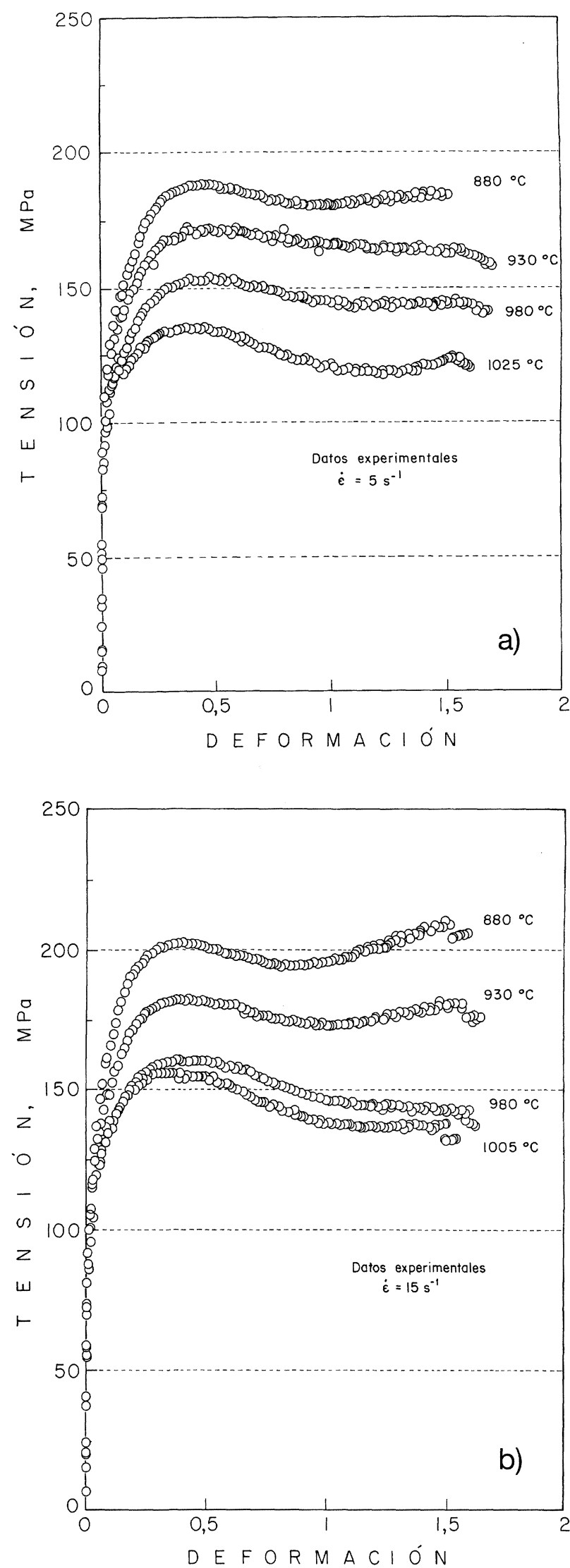

FIG. 7.- Datos experimentales de tensión-deformación: a) $5 \mathrm{~s}^{-1}$, y b) $15 \mathrm{~s}^{-1}$.

FIG. 7.- Stress-strain experimental data: a) $5 \mathrm{~s}^{-1}$, and $b) 15 \mathrm{~s}^{-1}$.
Los valores de los coeficientes que se van determinando para cada ecuación constituyen una nueva solución, en un proceso iterativo cuyo final viene marcado por el valor fijado para la convergencia del proceso.

En el presente trabajo se ha simplificado el proceso de cálculo al asignar valores a los coeficientes $n$ y $m$ de los tramos T1 y T2. No obstante, todo parece indicar que la elección de los valores de $n$ y $m$ no resulta trascendente en el ajuste de las curvas a los datos experimentales, habida cuenta de los efectos correspondientes que otros coeficientes objeto de estudio- tienen en dicho ajuste.

El método proporciona las curvas tensión-deformación (Figs. 8a) y 8b) de un acero microaleado con niobio y titanio, y posibilita la deducción de tensiones para otras características de la deformación, por interpolación con los que aquí se presen$\tan$.

\section{Agradecimientos}

Los autores agradecen al Prof. Sellars las facilidades para la utilización de los medios disponibles en el Laboratorio de Deformación en Caliente de la Universidad de Sheffield (R.U.), así como a la CECA (convenio 7210-EC/936-93-D3.01d) que ha hecho posible la fabricación y procesado del material y el soporte económico para la ejecución de los ensayos.

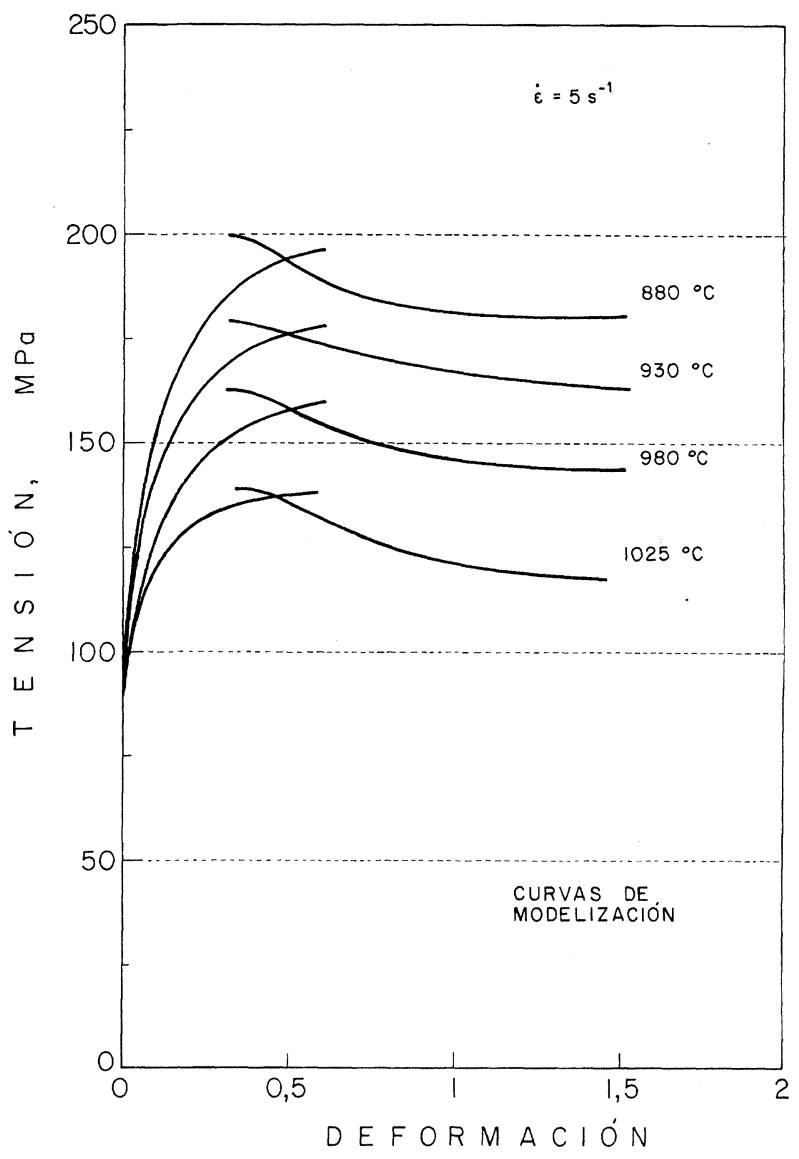

Rev. Metal. Madrid, 33 (1), 1997 


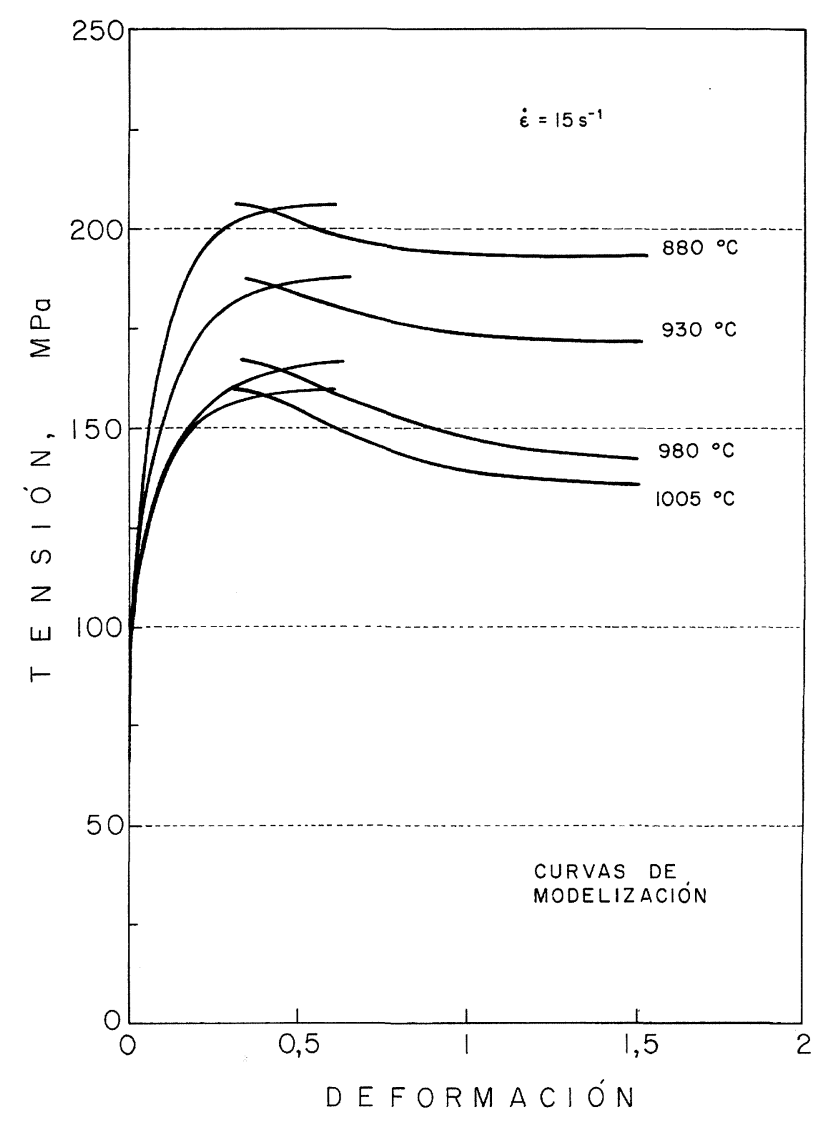

FIG. 8.- Curvas de modelización tensión-deformación: a) $5 \mathrm{~s}^{-1}$, y b) $15 \mathrm{~s}^{-1}$.

FIG. 8.- Stress-strain modeled curves: $a$ : $5 s^{-1}$, and b) $15 \mathrm{~s}^{-1}$.

\section{BIBLIOGRAFÍA}

- Sellars, C.M. Technology of Hot Flat Rolling (Vol. 1). "Microstructural Changes During Hot Working Operations". A Short Course for Engineering and Materials Graduates. Dept. Engineering of Materials. The University of Sheffield (R.U.). 11-23 Sept. 1988.

- Sellars, C.M. Technology of Hot Flat Rolling (Vol. 1). "Strength During Hot Working Opperations". A Short Course for Engineering and Materials Graduates. Dept. Engineering of Materials. The University of Sheffield (R.U.). 11-23 Sept. 1988.

- Puchi, E.S., McLaren, A.J. y Sellars, C.M. "Stress-strain Behaviour of Commercial Aluminium Alloys under HotWorking Conditions". Pendiente de publicación.

- Sah, J.P., Richardson, G.J. y Sellars, C.M. J. Aust. Inst. Met., 14: 292-297.

- Asensio, J. Trabajo no publicado. Dept. Engineering of Materials. The University of Sheffield (R.U.). 1993. 